\title{
EXTENSIONS OF POLYNOMIALS ON PREDUALS OF LORENTZ SEQUENCE SPACES
}

\author{
YUN SUNG CHOI, KWANG HEE HAN and HYUN GWI SONG \\ Department of Mathematics, Pohang University of Science and Technology, Pohang, 790-784, Korea \\ e-mail:mathchoi@postech.ac.kr,hankh@postech.ac.kr,hyuns@postech.ac.kr
}

(Received 24 November, 2004; accepted 11 April, 2005)

\begin{abstract}
We show that there is a unique norm-preserving extension for normattaining 2-homogeneous polynomials on the predual $d_{*}(w, 1)$ of a complex Lorentz sequence space $d(w, 1)$ to $d^{*}(w, 1)$, but there is no unique norm-preserving extension from $\mathcal{P}\left({ }^{n} d_{*}(w, 1)\right)$ to $\mathcal{P}\left({ }^{n} d^{*}(w, 1)\right)$ for $n \geq 3$.
\end{abstract}

2000 Mathematics Subject Classification. 46G25, 46A22.

1. Introduction. A bounded linear functional on a Banach space $E$ has clearly a norm preserving extension to its bidual $E^{* *}$ by the Hahn-Banach theorem. In particular, when the Banach space $E$ is an $M$-ideal in $E^{* *}$, the extension is unique.

Aron and Berner [2] first studied "Hahn-Banach type theorems" for spaces of polynomials on Banach spaces in 1978. They proved that every continuous $n$ homogeneous polynomial $P$ on a Banach space $E$ can be extended to a continuous $n$-homogeneous polynomial $\widehat{P}$ to its bidual $E^{* *}$. In 1989 Davie and Gamelin [4] showed that the Aron-Berner extension $\widehat{P}$ is a norm-preserving extension of $P$. These facts lead us to the following question. What classes of continuous $n$-homogeneous polynomials on a Banach space $E$ can have a unique norm-preserving extension to its bidual $E^{* *}$, when the Banach space $E$ is an $M$-ideal in $E^{* *}$ ? For example, $c_{0}$ and the predual $d_{*}(w, 1)$ of a Lorentz sequence space $d(w, 1)$ is an $M$-ideal in its bidual $l_{\infty}$ and $d^{*}(w, 1)$, respectively [5].

Aron, Boyd and Choi [3] proved that every norm-attaining 2-homogeneous polynomial on complex $c_{0}$ has a unique norm-preserving extension to $l_{\infty}$. They also showed that for $n \geq 3$ there exists a norm-attaining $n$-homogeneous polynomial on $c_{0}$ whose norm-preserving extension to $l_{\infty}$ is not unique. However, it is still an open problem whether every continuous 2 -homogeneous polynomial on complex $c_{0}$ has a unique norm-preserving extension. For real $c_{0}$ they showed that there exists a normattaining $n$-homogeneous polynomial on $c_{0}$ whose norm-preserving extension is not unique.

Since $d_{*}(w, 1)$ contains a subspace isomorphic to $c_{0}$, we became interested in the same problems on $d_{*}(w, 1)$ as studied on $c_{0}$ in [3]. Both cases show the same results about the uniqueness of norm-preserving extension, but there is a different property between those polynomials. The main results of this article are the following.

(1) In the real case, for $n \geq 2$ we construct an $n$-homogeneous polynomial on $d_{*}(w, 1)$ with two distinct norm-preserving extensions to its bidual $d^{*}(w, 1)$.

This research is supported in part by KOSEF Interdisciplinary Research Program Grant 1999-2-102-003-5 of Korea. 
(2) In the complex case, every norm-attaining 2-homogeneous polynomial on $d_{*}(w, 1)$ is finite, but this is not true for $n$-homogeneous polynomials, $n \geq 3$. Furthermore, we show that every norm-attaining 2-homogeneous polynomial on $d_{*}(w, 1)$ has a unique norm-preserving extension to $d^{*}(w, 1)$, but for $n \geq 3$ there exists a normattaining $n$-homogeneous polynomial whose norm-preserving extension is not unique.

(3) It was proved in [3] that if an $n$-homogeneous polynomial $P$ on $l_{\infty}$ satisfies $\|P\|=\left\|\left.P\right|_{c_{0}}\right\|$, then it is $w^{*}$-continuous on bounded sets at 0 . Differently from that, for $w \in l_{2} \backslash l_{1}$ there is an $n$-homogeneous polynomial $P$ on $d^{*}(w, 1)$ with $\|P\|=\left\|\left.P\right|_{d_{*}(w, 1)}\right\|$, but $P$ is not $w^{*}$-continuous on bounded sets at 0 .

2. Main results. Let $w=\left(w_{i}\right)_{i=1}^{\infty}$ be a decreasing sequence of positive numbers such that $w \in c_{0} \backslash l_{1}$, which is called an admissible sequence. Given a sequence $x=\left(x_{i}\right)$ of scalars, let $[x]=\left([x]_{i}\right)_{i=1}^{\infty}$ be the rearrangement of $\left(\left|x_{i}\right|\right)_{i=1}^{\infty}$ so that $[x]_{i} \geq[x]_{i+1}$ for all $i \in \mathbb{N}$. The Lorentz sequence space $d(w, 1)$ is defined to be the Banach space of all sequences of scalars $x=\left(x_{1}, x_{2}, \ldots\right)$ for which $\|x\|=\sum_{n=1}^{\infty}[x]_{n} w_{n}<\infty$. Recall that its dual space

$$
d^{*}(w, 1)=\left\{\left(x_{i}\right)_{i=1}^{\infty} ; \quad\left(\frac{\sum_{i=1}^{k}[x]_{i}}{\sum_{i=1}^{k} w_{i}}\right)_{k=1}^{\infty} \in l_{\infty}\right\}
$$

has the norm defined by

$$
\|x\|=\sup _{k} \frac{\sum_{i=1}^{k}[x]_{i}}{\sum_{i=1}^{k} w_{i}}, \quad x=\left(x_{k}\right)_{k=1}^{\infty} \in d^{*}(w, 1),
$$

and its predual space

$$
d_{*}(w, 1)=\left\{\left(x_{i}\right)_{i=1}^{\infty} ; \quad\left(\frac{\sum_{i=1}^{k}[x]_{i}}{\sum_{i=1}^{k} w_{i}}\right)_{k=1}^{\infty} \in c_{0}\right\}
$$

has the norm induced by $d^{*}(w, 1)$. Let $B_{E}$ be the closed unit ball of a Banach space $E$.

We can easily verify that $x=\left(x_{i}\right)_{i=1}^{\infty} \in B_{d^{*}(w, 1)}$ if and only if given a positive integer $n$

$$
\sum_{i \in I}\left|x_{i}\right| \leq \sum_{i=1}^{n} w_{i}
$$

for any finite subset $I=\left\{i_{1}, \ldots, i_{n}\right\}$ of $\mathbb{N}$.

Since $d_{*}(w, 1)$ is an $M$-ideal in $d^{*}(w, 1)$, it is clear that each $f \in\left(d_{*}(w, 1)\right)^{*}$ has a unique norm preserving extension $\tilde{f} \in\left(d^{*}(w, 1)\right)^{*}$. We now consider the problem of a unique norm-preserving extension for $n$-homogeneous polynomials on $d_{*}(w, 1)$ with $n \geq 2$.

In the real case, for $n \geq 2$ there exists a norm-attaining $n$-homogeneous polynomial on $d_{*}(w, 1)$ with two different norm-preserving extensions to $d^{*}(w, 1)$. To construct such polynomials let us first define a bounded linear operator $T: d^{*}(w, 1) \longrightarrow l_{\infty}$ by $T\left(\left(x_{i}\right)_{i=1}^{\infty}\right)=\left(y_{k}\right)_{k=1}^{\infty}$, where

$$
y_{k}=\frac{\sum_{i=1}^{k} x_{i}}{\sum_{i=1}^{k} w_{i}} \quad(k \in \mathbb{N}) .
$$

Clearly $T\left(d_{*}(w, 1)\right) \subset c_{0}, T(w)=(1,1,1, \ldots) \in \ell_{\infty}$ and $\|T\|=\|T(w)\|=1$. Let $\phi$ be a Banach limit functional on $l_{\infty}$, and define $\widetilde{\phi}=\phi \circ T \in\left(d^{*}(w, 1)\right)^{*}$. Since 
$\phi(x)=\lim _{i} x_{i}$ for a convergent sequence $x=\left(x_{i}\right) \in c$, we have that $\|\widetilde{\phi}\|=1, \widetilde{\phi}(w)=1$, and $\left.\widetilde{\phi}\right|_{d_{*}(w, 1)}=0$.

Now consider the $n$-homogeneous polynomial $P(x)=x_{1}{ }^{n}$ on $d_{*}(w, 1)$ with norm one. Then $P_{1}(x)=x_{1}{ }^{n}$ and $P_{2}(x)=x_{1}{ }^{n}-x_{1}{ }^{n-2} \widetilde{\phi}^{2}(x)$ are two distinct norm-preserving extensions of $P$ to its bidual $d^{*}(w, 1)$.

In the complex case, for $n \geq 3$ there also exists a norm-attaining $n$-homogeneous polynomial on $d_{*}(w, 1)$ with two distinct norm-preserving extensions to $d^{*}(w, 1)$. For this we need the following lemma.

Lemma 1. Suppose that $0<t<1,|\alpha| \leq 1,|\beta| \leq 1$, and $|\alpha|+|\beta| \leq 1+t$. For a positive integer $n \geq 3$,

$$
|t \alpha-\beta|^{n}+\left(1+t^{2}\right)|\alpha+t \beta|^{n-1} \leq\left(1+t^{2}\right)^{n} .
$$

Proof. For $0<t<1,|\alpha| \leq 1,|\beta| \leq 1$, and $|\alpha|+|\beta| \leq 1+t$, it is easily checked that $|t \alpha-\beta| \leq 1+t^{2},|\alpha|^{2}+|\bar{\beta}|^{2} \leq\left(1+t^{2}\right)$, and

$$
|t \alpha-\beta|^{2}+|\alpha+t \beta|^{2} \leq\left(1+t^{2}\right)\left(|\alpha|^{2}+|\beta|^{2}\right) \leq\left(1+t^{2}\right)^{2}
$$

Since $(a+b)^{p} \geq a^{p}+b^{p}$ for $a>0, b>0$ and $p \geq 1$,

$$
\begin{aligned}
|t \alpha-\beta|^{n}+\left(1+t^{2}\right)|\alpha+t \beta|^{n-1} & \leq\left(1+t^{2}\right)\left(|t \alpha-\beta|^{n-1}+|\alpha+t \beta|^{n-1}\right) \\
& \leq\left(1+t^{2}\right)\left(|t \alpha-\beta|^{2}+|\alpha+t \beta|^{2}\right)^{\frac{n-1}{2}} \leq\left(1+t^{2}\right)^{n} .
\end{aligned}
$$

Let an $n$-homogeneous polynomial $P$ on complex $d_{*}(w, 1)$ be defined by

$$
P(x)=\left(w_{2} x_{1}-x_{2}\right)^{n} .
$$

Clearly $\|P\|=\left(1+w_{2}^{2}\right)^{n}$ and $P$ attains its norm. Consider the following two $n$ homogeneous polynomials $P_{1}$ and $P_{2}$ on complex $d^{*}(w, 1)$ defined by

$$
P_{1}(x)=\left(w_{2} x_{1}-x_{2}\right)^{n}
$$

and

$$
P_{2}(x)=\left(w_{2} x_{1}-x_{2}\right)^{n}+\left(1+w_{2}^{2}\right)\left(x_{1}+w_{2} x_{2}\right)^{n-1} \widetilde{\phi}(x) .
$$

Clearly $\left.P_{1}\right|_{d_{*}(w, 1)}=P$, and $\left.P_{2}\right|_{d_{*}(w, 1)}=P$ because $\left.\widetilde{\phi}\right|_{d_{*}(w, 1)}=0$. It follows from Lemma 1 and the fact $\|\widetilde{\phi}\|=1$ that $\left\|P_{2}\right\| \leq\left(1+w_{2}^{2}\right)^{n}=P_{2}(w) \leq\left\|P_{2}\right\|$. Hence $\left\|P_{1}\right\|=\|P\|=\left\|P_{2}\right\|$. Note that $P_{1}(w)=0$, which implies that $P_{1}$ and $P_{2}$ are distinct norm preserving extensions of $P$ to $d^{*}(w, 1)$. Therefore, for $n \geq 3$ there exists a norm-attaining $n$ homogeneous polynomial on complex $d_{*}(w, 1)$ with two distinct norm-preserving extensions to $d^{*}(w, 1)$.

We recall that a 2 -homogeneous polynomial $P$ on $d_{*}(w, 1)$ is called finite if there exists a positive integer $n$ such that

$$
P(x)=\sum_{i=1}^{n}\left(\sum_{j=1}^{i} a_{i j} x_{i} x_{j}\right),
$$

for all $x=\left(x_{i}\right)_{i=1}^{\infty} \in d_{*}(w, 1)$. We note that the closed unit ball of $d_{*}(w, 1)$ has no extreme points, like $c_{0}$. See Lemma 2 of [1]. 
TheOREM 2. A 2-homogeneous polynomial P on complex $d_{*}(w, 1)$ attains its norm if and only if it is finite.

Proof. If $P$ is a finite 2-homogeneous polynomial on $d_{*}(w, 1)$, it can be regarded as a polynomial defined on an $n$-dimensional subspace of $d_{*}(w, 1)$ for some $n \in \mathbb{N}$ and hence $P$ attains its norm.

Conversely, suppose a 2-homogeneous polynomial $P$ attains its norm at $x_{0}=$ $\left(\lambda_{i}\right)_{i=1}^{\infty} \in B_{d_{*}(w, 1)}$. Without loss of generality we may assume that $\|P\|=1=P\left(x_{0}\right)$. By change of variable and rearrangement of indices, we may assume that $x_{0}=\left(\lambda_{i}\right)_{i=1}^{\infty}$ satisfies $\lambda_{i} \geq \lambda_{i+1}, \lambda_{i} \geq 0$ for all $i \in \mathbb{N}$. Obviously

$$
\left\|x_{0}\right\|=\sup _{k} \frac{\sum_{i=1}^{k} \lambda_{i}}{\sum_{i=1}^{k} w_{i}}=1
$$

Since

$$
\lim _{k \rightarrow \infty} \frac{\sum_{i=1}^{k} \lambda_{i}}{\sum_{i=1}^{k} w_{i}}=0
$$

we can choose the largest positive integer $n$ such that

$$
\sum_{i=1}^{n} \lambda_{i}=\sum_{i=1}^{n} w_{i} \quad \text { and } \quad \sum_{i=1}^{k} \lambda_{i}<\sum_{i=1}^{k} w_{i} \text { for all } k \geq n+1 .
$$

Let

$$
a=1-\sup \left\{\frac{\sum_{i=1}^{k} \lambda_{i}}{\sum_{i=1}^{k} w_{i}} ; k \geq n+1\right\}>0 .
$$

Clearly $\lambda_{n}>\lambda_{n+1}$. Choose $\delta>0$ such that $\lambda_{n}>\lambda_{n+1}+\delta$ and let

$$
b=\min \{a, \delta\}>0 .
$$

Let $y=\left(0, \ldots, 0, y_{n+1}, y_{n+2}, \ldots\right) \in B_{d_{*}(w, 1)}$ and $\lambda$ with $|\lambda| \leq b$ be given. Then we have

$$
x_{0}+\lambda y=\left(\lambda_{1}, \ldots, \lambda_{n}, \lambda_{n+1}+\lambda y_{n+1}, \lambda_{n+2}+\lambda y_{n+2}, \ldots\right)
$$

and

$$
\lambda_{1} \geq \lambda_{2} \geq \cdots \geq \lambda_{n}>\lambda_{n+1}+\delta \geq \lambda_{i}+\left|\lambda \| y_{i}\right| \geq\left|\lambda_{i}+\lambda y_{i}\right|
$$

for all $i \geq n+1$.

Let $\overline{\mathbb{N}}_{0}=\mathbb{N} \backslash\{1,2, \ldots, n\}$. Given $k \geq n+1$ and a finite subset $J$ of $\mathbb{N}_{0}$ with $|J|=$ $k-n$, we have

$$
\begin{aligned}
\sum_{i=1}^{n} \lambda_{i}+\sum_{i \in J}\left|\lambda_{i}+\lambda y_{i}\right| & \leq \sum_{i=1}^{n} \lambda_{i}+\sum_{i \in J} \lambda_{i}+a \sum_{i=1}^{k-n} w_{i} \\
& \leq \sum_{i=1}^{n} \lambda_{i}+\sum_{i \in J} \lambda_{i}+\left(1-\frac{\sum_{i=1}^{k} \lambda_{i}}{\sum_{i=1}^{k} w_{i}}\right) \sum_{i=1}^{k} w_{i} \\
& =\sum_{i=1}^{k} w_{i}+\left(\sum_{i \in J} \lambda_{i}-\sum_{i=n+1}^{k} \lambda_{i}\right) \leq \sum_{i=1}^{k} w_{i}
\end{aligned}
$$


which implies that

$$
x_{0}+\lambda y \in B_{d_{*}(w, 1)} .
$$

Hence we obtain

$$
\left|P\left(x_{0} \pm \lambda y\right)\right|=\left|1 \pm 2 \lambda \breve{P}\left(x_{0}, y\right)+\lambda^{2} P(y)\right| \leq\left|P\left(x_{0}\right)\right|=1,
$$

where $\breve{P}$ is the unique symmetric bilinear form associated with $P$. It follows from a phase manipulation that

$$
P(y)=0, \quad \breve{P}\left(x_{0}, y\right)=0 .
$$

Taking $y_{0}=\left(0, \ldots, 0, \lambda_{n+1}, \lambda_{n+2}, \ldots\right)$ we clearly have

$$
P\left(y_{0}\right)=0, \quad \breve{P}\left(x_{0}, y_{0}\right)=0,
$$

which implies that

$$
P\left(\lambda_{1}, \lambda_{2}, \ldots, \lambda_{n}, 0, \ldots\right)=P\left(x_{0}-y_{0}\right)=P\left(x_{0}\right)+P\left(y_{0}\right)-2 \breve{P}\left(x_{0}, y_{0}\right)=P\left(x_{0}\right)=1 .
$$

Define

$$
\begin{aligned}
z_{1} & =\left(\lambda_{1}, \lambda_{2}, \ldots, \lambda_{n}\right) \\
z_{2} & =\left(\lambda_{1}, \lambda_{2}-n \lambda_{2}, \ldots, \lambda_{n}\right) \\
& \vdots \\
z_{n} & =\left(\lambda_{1}, \lambda_{2}, \ldots, \lambda_{n}-n \lambda_{n}\right) .
\end{aligned}
$$

Repeating the argument given above we see that $\breve{P}\left(\tilde{z}_{1}, y\right)=0$, where $\tilde{z}_{j}=\left(z_{j}, 0,0, \ldots\right)$, $j=1, \ldots, n$. Since

$$
\left(x_{1}, x_{2}, \ldots, x_{n}\right)=\frac{1}{n}\left(\frac{x_{1}}{\lambda_{1}}+\frac{x_{2}}{\lambda_{2}}+\cdots+\frac{x_{n}}{\lambda_{n}}\right) z_{1}+\frac{1}{n} \sum_{j=2}^{n}\left(\frac{x_{1}}{\lambda_{1}}-\frac{x_{j}}{\lambda_{j}}\right) z_{j},
$$

we have

$$
P\left(x_{1}, x_{2}, \ldots, x_{n}, y_{n+1}, y_{n+2}, \ldots\right)=P\left(x_{1}, x_{2}, \ldots, x_{n}, 0, \ldots\right)+\frac{2}{n} \sum_{j=2}^{n}\left(\frac{x_{1}}{\lambda_{1}}-\frac{x_{j}}{\lambda_{j}}\right) \breve{P}\left(\tilde{z}_{j}, y\right) .
$$

Applying the same computation as in Proposition 2 in [3] we have $\breve{P}\left(\tilde{z}_{j}, y\right)=0$, for all $j, 2 \leq j \leq n$ and hence $P$ depends only on finitely many variables $x_{1}, x_{2}, \ldots, x_{n}$.

REMARK 3. Sevilla and Payá [6] proved that every norm-attaining $n$-homogeneous polynomial $P$ on complex $d_{*}(w, 1)$ satisfies $P\left(e_{k}\right)=0$, for sufficiently large $k \in \mathbb{N}$, where $\left\{e_{k}\right\}_{k=1}^{\infty}$ is the standard unit vector basis of $d_{*}(w, 1)$. Theorem 2 is stronger than this for 2-homogeneous polynomials.

REMARK 4 . For $n \geq 3$, there exists a norm attaining $n$-homogeneous polynomial $P$ on $d_{*}(w, 1)$ that is not finite. Let

$$
P(x)=\left(w_{2} x_{1}-x_{2}\right)^{n}+\left(1+w_{2}^{2}\right)\left(x_{1}+w_{2} x_{2}\right)^{n-1} \sum_{j=3}^{\infty} \frac{x_{j}}{2^{j}} .
$$


Clearly $P$ is not finite. By Lemma 1 we have

$$
|P(x)| \leq\left|w_{2} x_{1}-x_{2}\right|^{n}+\left(1+w_{2}^{2}\right)\left|x_{1}+w_{2} x_{2}\right|^{n-1} \leq\left(1+w_{2}^{2}\right)^{n} .
$$

Since $\left|P\left(x_{0}\right)\right|=\left(1+w_{2}^{2}\right)^{n}$ for $x_{0}=\left(w_{2},-1,0,0, \ldots\right) \in B_{d_{*}(w, 1)}, P$ attains its norm.

THEOREM 5. Every 2-homogeneous norm-attaining polynomial on complex $d_{*}(w, 1)$ has a unique norm-preserving extension to $d^{*}(w, 1)$.

Proof. Suppose that $P$ is a norm-attaining 2-homogeneous polynomial on $d_{*}(w, 1)$ that attains its norm at $x_{0}=\left(\lambda_{i}\right)_{i=1}^{\infty} \in B_{d_{*}(w, 1)}$ and suppose that $Q$ is its norm-preserving extension to $d^{*}(w, 1)$. Let $n$ be the largest positive integer such that

$$
\sum_{i=1}^{n} \lambda_{i}=\sum_{i=1}^{n} w_{i} \quad \text { and } \quad \sum_{i=1}^{k} \lambda_{i}<\sum_{i=1}^{k} w_{i} \text { for all } k \geq n+1 .
$$

As in the proof of Theorem 2, we may assume that $P$ depends only on the first $n$ variables $x_{1}, \ldots, x_{n}$ and also that $\|P\|=1=P\left(z_{0}\right)$ for some $z_{0}=\left(\lambda_{1}, \lambda_{2}, \ldots, \lambda_{n}, 0,0, \ldots\right)$ with $\lambda_{1} \geq \lambda_{2} \geq \ldots \geq \lambda_{n}>0$. Let

$$
a=\min \left\{\lambda_{n}, 1-\frac{\sum_{i=1}^{n} \lambda_{i}}{\sum_{i=1}^{n+1} w_{i}}\right\}>0 .
$$

Let $y=\left(0, \ldots, 0, y_{n+1}, y_{n+2}, \ldots\right) \in B_{d^{*}(w, 1)}$ and let $\lambda$ with $|\lambda| \leq a$ be given. Then we have

$$
z_{0}+\lambda y=\left(\lambda_{1}, \lambda_{2}, \ldots, \lambda_{n}, \lambda y_{n+1}, \lambda y_{n+2}, \ldots\right)
$$

and

$$
\lambda_{1} \geq \lambda_{2} \cdots \geq \lambda_{n} \geq a \geq\left|\lambda y_{i}\right|,
$$

for all $i \geq n+1$. Let $\mathbb{N}_{0}=\mathbb{N} \backslash\{1,2, \ldots, n\}$. Given $k \geq n+1$ and a finite subset $J$ of $\mathbb{N}_{0}$ with $|J|=k-n$, we have

$$
\begin{aligned}
\sum_{i=1}^{n} \lambda_{i}+\sum_{i \in J}\left|\lambda y_{i}\right| & \leq \sum_{i=1}^{n} \lambda_{i}+a \sum_{i=1}^{k-n} w_{i} \\
& \leq \sum_{i=1}^{n} \lambda_{i}+\left(1-\frac{\sum_{i=1}^{n} \lambda_{i}}{\sum_{i=1}^{k} w_{i}}\right) \sum_{i=1}^{k} w_{i} \\
& =\sum_{i=1}^{k} w_{i}
\end{aligned}
$$

which implies that

$$
z_{0}+\lambda y \in B_{d^{*}(w, 1)}
$$

Let $\breve{Q}$ be the unique symmetric bilinear form associated with $Q$. Since

$$
\left|Q\left(z_{0} \pm \lambda y\right)\right|=\left|1 \pm 2 \lambda \breve{Q}\left(z_{0}, y\right)+\lambda^{2} Q(y)\right| \leq\left|Q\left(z_{0}\right)\right|=1,
$$


we can see that $Q(y)=0, \breve{Q}\left(z_{0}, y\right)=0$, for all $y=\left(0, \ldots, 0, y_{n+1}, \ldots\right) \in B_{d^{*}(w, 1)}$. As in the proof of Theorem 2, we conclude again that $Q$ depends only on the first $n$ variables. If $Q_{1}$ and $Q_{2}$ are norm-preserving extensions of $P$ to $d^{*}(w, 1)$, then

$$
Q_{1}\left(x_{1}, \ldots, x_{n}, x_{n+1}, \ldots\right)=P\left(x_{1}, \ldots, x_{n}, 0, \ldots\right)=Q_{2}\left(x_{1}, \ldots, x_{n}, x_{n+1}, \ldots\right),
$$

for all $x=\left(x_{n}\right)_{n=1}^{\infty} \in d^{*}(w, 1)$. Hence $P$ has a unique norm-preserving extension to $d^{*}(w, 1)$.

We can see that $n$-homogeneous polynomials on $d_{*}(w, 1)$ have the same properties concerning the uniqueness of norm-preserving extensions as those on $c_{0}$. However, they don't always share the same properties as polynomials on $c_{0}$. For instance, every continuous polynomial on $c_{0}$ is weakly continuous on bounded sets and Proposition 4 in [3] shows that every $n$-homogeneous polynomial $P$ on $l_{\infty}$ with $\|P\|=\left\|\left.P\right|_{c_{0}}\right\|$ is $w^{*}$-continuous on bounded sets at 0 . In Example 7 we can find a continuous 2homogeneous polynomial $P$ on $d^{*}(w, 1)$ such that $\|P\|=\left\|\left.P\right|_{d_{*}(w, 1)}\right\|$, but $P$ is not $w^{*}$-continuous on bounded sets at 0 and $\left.P\right|_{d_{*}(w, 1)}$ is not weakly continuous on bounded sets at 0 .

LEMMA 6. Let $\left(x_{i}\right)$ and $\left(y_{i}\right)$ be decreasing sequences of nonnegative real numbers. If $\sum_{i=1}^{n} y_{i} \leq \sum_{i=1}^{n} x_{i}$ for every positive integer $n$, then $\sum_{i=1}^{n} y_{i}^{2} \leq \sum_{i=1}^{n} x_{i}^{2}$ for every positive integer $n$.

Proof. We are going to prove the result by induction. It is clear for $n=1$. Suppose that it is true for the positive integer $n=k-1$. If $y_{k} \leq x_{k}$, clearly $\sum_{i=1}^{k} y_{i}^{2} \leq \sum_{i=1}^{k} x_{i}^{2}$ by the induction hypothesis; hence we might as well assume $x_{k}<y_{k}$. Let $J=\left\{j: x_{j}<\right.$ $\left.y_{j}, 1<j \leq k\right\}$. If the cardinality of the set $J$ is $l$, then we write $J=\left\{j_{1}<j_{2}<\cdots<\right.$ $\left.j_{l}=k\right\}$. Put $\alpha_{i}=x_{i}-y_{i} \geq 0$ for $i \notin J, 1 \leq i<k$, and $\beta_{j}=y_{j}-x_{j}>0$ for $j \in J$. Since $\sum_{i=1}^{n} y_{i} \leq \sum_{i=1}^{n} x_{i}$ for every positive integer $1 \leq n \leq k$, we have

$$
\begin{aligned}
& \beta_{j_{1}} \leq \alpha_{1}+\cdots+\alpha_{j_{1}-1}, \\
& \beta_{j_{1}}+\beta_{j_{2}} \leq\left(\alpha_{1}+\cdots+\alpha_{j_{1}-1}\right)+\left(\alpha_{j_{1}+1}+\cdots+\alpha_{j_{2}-1}\right), \\
& \vdots \\
& \sum_{j \in J} \beta_{j} \leq \sum_{i=1, i \notin J}^{k-1} \alpha_{i} .
\end{aligned}
$$

Therefore,

$$
\begin{aligned}
\sum_{i=1}^{k} x_{i}^{2}= & {\left[\sum_{i=1}^{j_{1}-1}\left(y_{i}+\alpha_{i}\right)^{2}+\left(y_{j_{1}}-\beta_{j_{1}}\right)^{2}\right]+\left[\sum_{i=j_{1}+1}^{j_{2}-1}\left(y_{i}+\alpha_{i}\right)^{2}+\left(y_{j_{2}}-\beta_{j_{2}}\right)^{2}\right] } \\
& +\cdots+\left[\sum_{i=j_{l-1}+1}^{k-1}\left(y_{i}+\alpha_{i}\right)^{2}+\left(y_{k}-\beta_{k}\right)^{2}\right] \\
\geq & \sum_{i=1}^{k} y_{i}^{2}+2\left\{\left(\sum_{i=1}^{j_{1}-1} \alpha_{i}\right)-\beta_{j_{1}}\right\} y_{j_{1}}+2\left\{\left(\sum_{i=j_{1}+1}^{j_{2}-1} \alpha_{i}\right)-\beta_{j_{2}}\right\} y_{j_{2}} \\
& +\cdots+2\left\{\left(\sum_{i=j_{l-1}+1}^{k-1} \alpha_{i}\right)-\beta_{k}\right\} y_{k}
\end{aligned}
$$




$$
\begin{aligned}
& \geq \sum_{i=1}^{k} y_{i}^{2}+2\left\{\left(\sum_{i=1, i \neq j_{1}}^{j_{2}-1} \alpha_{i}\right)-\left(\beta_{j_{1}}+\beta_{j_{2}}\right)\right\} y_{j_{2}}+\cdots+2\left\{\left(\sum_{i=j_{l-1}+1}^{k-1} \alpha_{i}\right)-\beta_{k}\right\} y_{k} \\
& \vdots \\
& \geq \sum_{i=1}^{k} y_{i}^{2}+2\left\{\left(\sum_{i=1, i \notin J}^{k-1} \alpha_{i}\right)-\left(\sum_{j \in J} \beta_{j}\right)\right\} y_{k} \geq \sum_{i=1}^{k} y_{i}^{2},
\end{aligned}
$$

where the inequalities follow from the above inequalities and the fact that the sequence $\left(y_{i}\right)$ is decreasing.

EXAmPLE 7. Let $w=\left(w_{i}\right)_{i=1}^{\infty} \in l_{2} \backslash l_{1}$ and define the 2-homogeneous polynomial $P$ on $d^{*}(w, 1)$ by

$$
P(x)=\sum_{i=1}^{\infty} x_{i}^{2}, \quad x=\left(x_{i}\right)_{i=1}^{\infty} \in d^{*}(w, 1) .
$$

It follows from Lemma 6 that $\|P\|=\sum_{i=1}^{\infty} w_{i}^{2}=\left\|\left.P\right|_{d_{*}(w, 1)}\right\|$. However, the sequence $\left(e_{i}\right)_{i=1}^{\infty}$ converges weak-star (weakly) to 0 in $d^{*}(w, 1)\left(d_{*}(w, 1)\right)$, and $P\left(e_{i}\right)=1$ for all $i$. Therefore, $P$ is not $w^{*}$-continuous on bounded sets at 0 , and $\left.P\right|_{d_{*}(w, 1)}$ is not weakly continuous on bounded sets at 0 .

Let $\mathbf{i}^{n}=\left(i_{1}, \ldots, i_{n}\right) \in \mathbb{N}^{n}$. We denote by $B_{\mathbf{i}^{n}}$ the closed unit ball of the $n$-dimensional subspace of $d^{*}(w, 1)$ spanned by $\left\{e_{i_{1}}, \ldots, e_{i_{n}}\right\}$. By the Krein-Milman theorem, $B_{\mathbf{i}^{n}}$ is the (closed) convex hull of its extreme points, that is, $B_{\mathbf{i}^{n}}=\operatorname{co}\left(\operatorname{ext}\left(B_{\mathbf{i}^{n}}\right)\right)$. It is worthwhile to characterize its extreme points.

Proposition 8. Given $\mathbf{i}^{n}=\left(i_{1}, \ldots, i_{n}\right) \in \mathbb{N}^{n}$, the extreme points $\left(x_{i}\right)$ of $B_{\mathbf{i}^{n}}$ are the points with coordinates $\left|x_{i_{j}}\right|=w_{\sigma(j)}, 1 \leq j \leq n$, for some permutation $\sigma$ on $\{1,2, \ldots, n\}$ and $x_{i}=0$, otherwise.

Proof. We might as well assume $\mathbf{i}^{n}=(1, \ldots, n)$. An easy computation shows that the points with coordinates $\left|x_{i}\right|=w_{\sigma(i)}, 1 \leq i \leq n$ for some permutation $\sigma$ on $\{1,2, \ldots, n\}$ and $x_{i}=0$ otherwise, are extreme points of $B_{\mathrm{i}^{n}}$.

We shall prove that the other points $x$ in $B_{\mathbf{i}^{n}}$ are not extreme points. Without loss of generality we may assume that $x=\left(x_{i}\right)$ is rearranged so that $\left|x_{1}\right| \geq\left|x_{2}\right| \geq \cdots \geq\left|x_{n}\right|$. Let $k$ be the smallest positive integer $i$ with $\left|x_{i}\right| \neq w_{i}$. If $k=n$, then $\left|x_{n-1}\right|=w_{n-1}>w_{n}>$ $\left|x_{n}\right|$. Choose $\delta>0$ so that $\left|x_{n}\right|+\delta<w_{n}$. Set $u$ and $v$ to be the points in $B_{\mathbf{i}^{n}}$ such that

$$
u=\left(x_{1}, \ldots, x_{n-1}, \operatorname{sgn}\left(x_{n}\right)\left(\left|x_{n}\right|+\delta\right)\right)
$$

and

$$
v=\left(x_{1}, \ldots, x_{n-1}, \operatorname{sgn}\left(x_{n}\right)\left(\left|x_{n}\right|-\delta\right)\right) .
$$

Then $x=1 / 2(u+v)$, and hence $x$ is not an extreme points.

Suppose that $1<k<n$. Let $p=\max \left\{l:\left|x_{l}\right|=\left|x_{k}\right|, k \leq l \leq n\right\}$. If $p=k$, then $\left|x_{k-1}\right|=w_{k-1}>w_{k}>\left|x_{k}\right|>\left|x_{k+1}\right|$. Let $q=\max \left\{l:\left|x_{l}\right|=\left|x_{k+1}\right|, k+1 \leq l \leq n\right\}$. If $q<n$, choose $\delta>0$ so that $w_{k}>\left|x_{k}\right|+\delta,\left|x_{k}\right|-\delta>\left|x_{k+1}\right|+\delta,\left|x_{q}\right|-\delta>\left|x_{q+1}\right|$ and

$$
\left|x_{k}\right|+\left|x_{k+1}\right|+\cdots+\left|x_{k+j}\right|+\delta<w_{k}+w_{k+1}+\cdots+w_{k+j},
$$


for all $j, 1 \leq j \leq q-k-1$. We note that

$$
\left|x_{k}\right|+\left|x_{k+1}\right|+\cdots+\left|x_{k+j}\right|<w_{k}+w_{k+1}+\cdots+w_{k+j},
$$

for all $j, 1 \leq j \leq q-k-1$. In the case where $q=n$, the condition $\left|x_{q}\right|-\delta>\left|x_{q+1}\right|$ is omitted for the choice of $\delta$. Set $u=\left(u_{i}\right)$ and $v=\left(v_{i}\right)$ to be the points in $B_{\mathbf{i}^{n}}$ such that

$$
\begin{aligned}
& u_{k}=\operatorname{sgn}\left(x_{k}\right)\left(\left|x_{k}\right|-\delta\right), \quad v_{k}=\operatorname{sgn}\left(x_{k}\right)\left(\left|x_{k}\right|+\delta\right), \\
& u_{k+1}=\operatorname{sgn}\left(x_{k+1}\right)\left(\left|x_{k+1}\right|+\delta\right), \quad v_{k+1}=\operatorname{sgn}\left(x_{k+1}\right)\left(\left|x_{k+1}\right|-\delta\right)
\end{aligned}
$$

and $u_{i}=x_{i}=v_{i}$ for $i \neq k, k+1$. Then $x=(u+v) / 2$, and hence $x$ is not an extreme point.

If $k<p<n$, choose $\delta>0$ so that $w_{k}>\left|x_{k}\right|+\delta,\left|x_{p}\right|-\delta>\left|x_{p+1}\right|$ and

$$
\left|x_{k}\right|+\left|x_{k+1}\right|+\cdots+\left|x_{k+j}\right|+\delta<w_{k}+w_{k+1}+\cdots+w_{k+j}
$$

for all $j, 1 \leq j \leq p-k-1$. In the case where $p=n$, the condition $\left|x_{p}\right|-\delta>\left|x_{p+1}\right|$ is omitted for the choice of $\delta$. Set $u=\left(u_{i}\right)$ and $v=\left(v_{i}\right)$ to be the points in $B_{\mathbf{i}^{n}}$ such that

$$
\begin{array}{ll}
u_{k}=\operatorname{sgn}\left(x_{k}\right)\left(\left|x_{k}\right|+\delta\right), & v_{k}=\operatorname{sgn}\left(x_{k}\right)\left(\left|x_{k}\right|-\delta\right), \\
u_{p}=\operatorname{sgn}\left(x_{p}\right)\left(\left|x_{p}\right|-\delta\right), & v_{p}=\operatorname{sgn}\left(x_{p}\right)\left(\left|x_{p}\right|+\delta\right)
\end{array}
$$

and $u_{i}=x_{i}=v_{i}$ for $i \neq k$, $p$. Then $x=(u+v) / 2$, and hence $x$ is not an extreme point.

By a similar argument to the above the same conclusion can be drawn for the case remaining where $k=1$.

The proof of Lemma 6 also follows from Proposition 8. Given a positive integer $n$, let $\mathbf{i}^{n}=(1, \ldots, n)$ and $\left(w_{1}, \ldots, w_{n}\right)=\left(x_{1}, \ldots, x_{n}\right)$. Then $y=\left(y_{1}, \ldots, y_{n}\right) \in B_{\mathbf{i}^{n}}$, and it is a convex combination of extreme points of $B_{\mathbf{i}^{n}}$. For simplicity, suppose that $y=\lambda e_{1}+(1-\lambda) e_{2}$, where $0 \leq \lambda \leq 1$ and $e_{k}=\left(w_{\sigma_{k}(j)}\right)_{j=1}^{n}$, for some permutation $\sigma_{k}$ on $\{1,2, \ldots, n\}, k=1,2$. Then $\sum_{i=1}^{n} y_{i}^{2}=a \lambda^{2}+b \lambda+c$ for some real numbers $a>0, b$ and $c$. Since it is always positive on the interval $0 \leq \lambda \leq 1$ and $a>0$, its maximum on $0 \leq \lambda \leq 1$ occurs at $\lambda=0$ or 1 . Therefore, $\sum_{i=1}^{n} y_{i}^{2} \leq \sum_{i=1}^{n} w_{i}^{2}$.

\section{REFERENCES}

1. M. D. Acosta, F. J. Aguirre and R. Payá, There is no bilinear Bishop-Phelps theorem, Israel J. Math. 93 (1996), 221-227.

2. R. M. Aron and P. Berner, A Hahn-Banach extension theorem for analytic mappings, Bull. Math. Soc. France 106 (1978), 3-24.

3. R. M. Aron, C. Boyd and Y. S. Choi, Unique Hahn-Banach theorems for spaces of homogeneous polynomials, J. Austral. Math. Soc. 70 (2001), 387-400.

4. A. M. Davie and T. W. Gamelin, A theorem on polynomial-star approximation, Proc. Amer. Math. Soc. 106 (1989), 351-356.

5. P. Harmand, D. Werner and W. Werner, $M$-ideals in Banach spaces and Banach algebras, Lecture Notes in Mathematics No. 1547 (Springer-Verlag, 1993).

6. M. J. Sevilla and R. Payá, Norm attaining multilinear forms and polynomials on preduals of Lorentz sequence spaces, Studia Math. 127 (1998), 99-112. 